OPEN ACCESS

Edited by:

Gianni Ciofani

Politecnico di Torino, Italy

Reviewed by:

Ciro Chiappini,

King's College London,

United Kingdom

Vincenzo Calcagno,

Universität Leipzig, Germany

Stefano Leporatti,

Istituto di

Nanotecnologia (NANOTEC), Italy

*Correspondence:

Stefano Luin

s.luin@sns.it

Specialty section:

This article was submitted to

Nanobiotechnology,

a section of the journal

Frontiers in Bioengineering and

Biotechnology

Received: 14 October 2018 Accepted: 06 November 2018 Published: 27 November 2018

Citation:

Cervadoro A, Palomba R, Vergaro $G$ Cecchi R, Menichetti L, Decuzzi P, Emdin M and Luin S (2018) Targeting Inflammation With Nanosized Drug Delivery Platforms in Cardiovascular Diseases: Immune Cell Modulation in

Atherosclerosis.

Front. Bioeng. Biotechnol. 6:177. doi: 10.3389/fbioe.2018.00177
Targeting Inflammation With Nanosized Drug Delivery Platforms in Cardiovascular Diseases: Immune Cell Modulation in Atherosclerosis

\author{
Antonio Cervadoro ${ }^{1}$, Roberto Palomba ${ }^{2}$, Giuseppe Vergaro ${ }^{3,4}$, Roberta Cecchi ${ }^{1,5}$, \\ Luca Menichetti ${ }^{6}$, Paolo Decuzzi ${ }^{2}$, Michele Emdin ${ }^{3,4}$ and Stefano Luin ${ }^{1,7 *}$ \\ ${ }^{1}$ NEST Laboratory, Scuola Normale Superiore, Pisa, Italy, ${ }^{2}$ Laboratory of Nanotechnology for Precision Medicine, \\ Fondazione Istituto Italiano di Tecnologia, Genova, Italy, ${ }^{3}$ Division of Cardiology and Cardiovascular Medicine, Fondazione \\ Toscana Gabriele Monasterio, Pisa, Italy, ${ }^{4}$ Institute of Life Sciences, Scuola Superiore Sant'Anna, Pisa, Italy, ${ }^{5}$ Center for \\ Nanotechnology Innovation (CNI@NEST), Istituto Italiano di Tecnologia, Pisa, Italy, ${ }^{6}$ CNR Institute of Clinical Physiology (IFC), \\ Pisa, Italy, ${ }^{7}$ NEST Laboratory, Istituto Nanoscienze, CNR, Pisa, Italy
}

Atherosclerosis (AS) is a disorder of large and medium-sized arteries; it consists in the formation of lipid-rich plaques in the intima and inner media, whose pathophysiology is mostly driven by inflammation. Currently available interventions and therapies for treating atherosclerosis are not always completely effective; side effects associated with treatments, mainly caused by immunodepression for anti-inflammatory molecules, limit the systemic administration of these and other drugs. Given the high degree of freedom in the design of nanoconstructs, in the last decades researchers have put high effort in the development of nanoparticles (NPs) formulations specifically designed for either drug delivery, visualization of atherosclerotic plaques, or possibly the combination of both these and other functionalities. Here we will present the state of the art of these subjects, the knowledge of which is necessary to rationally address the use of NPs for prevention, diagnosis, and/or treatment of AS. We will analyse the work that has been done on: (a) understanding the role of the immune system and inflammation in cardiovascular diseases, (b) the pathological and biochemical principles in atherosclerotic plaque formation, (c) the latest advances in the use of NPs for the recognition and treatment of cardiovascular diseases, (d) the cellular and animal models useful to study the interactions of NPs with the immune system cells.

Keywords: atherosclerosis, inflammatory diseases, smart nanomaterials, drug delivery, nanomedicine, imaging and theranostics, immune cells, cardiovascular diseases

\section{ATHEROSCLEROSIS AND INFLAMMATION}

Atherosclerotic disease, or simply atherosclerosis (AS), is initially characterized by the formation of fatty streaks, with the accumulation of lipids [primarily cholesterol, but also triglycerides (Goldberg, 2018)] in the intima and inner media of arterial wall, especially in regions with abnormal flow patterns (Chistiakov et al., 2017). Fatty streaks may then evolve into soft, lipid-rich plaques, and eventually into thick cap-calcified lesions and/or unstable plaques characterized by inflammatory infiltration and sustained oxidative processes. Stable fibrocalcific atheroma is characterized by 
calcium deposits, small lipid deposits, slight lumen reduction, and, often, by poor functional relevance. Vulnerable atheroma, which is more prone to rupture, is characterized by a large lipid-rich necrotic core, thin fibrous cap $(<65 \mu \mathrm{m})$, neovascularization, spotty calcifications, inflammatory cells, and positive remodeling (Moore and Tabas, 2011; Hansson et al., 2015). The possibility of plaque regression has been reviewed in Chistiakov et al. (2017) focusing on animal models, and in Fisher (2016) also considering the dynamic changes in lipid and immune cells distributions.

AS is currently considered an inflammatory disorder, characterized by the infiltration into sub-endothelial space of various immune cells (ICs), especially circulating monocytes (MCs) that subsequently differentiate into macrophages (MФs) and then into foam cells (FCs), going along with plaque formation and evolution (Moore et al., 2013; Zhang et al., 2017). Such simplified picture is complicated by the heterogeneity of the cells within and close to lesions during evolution [MCs, MФs, and FCs, but also neutrophils, dendritic cells (Butcher and Galkina, 2012), T cells (Taleb, 2016), and possibly others]. In the cited reviews particular attention is given to the markers of the various IC phenotypes, although cells can present more than one function and can express a continuum of markers of different subtypes (Butcher and Galkina, 2012).

A key example regards $M \Phi s$, the characterizing cells in AS: their different subtypes can have antithetic roles. Initially classified only as classically activated M1 (pro-inflammatory) or alternatively activated M2 (anti-inflammatory), evidences have brought to the definitions of additional subtypes (e.g., Mox, M4, Mhem) and even "sub-subtypes" (e.g., M2a, M2b, M2c); there could actually be a continuum of specializations, and $M \Phi$ s could even convert into each other (Butcher and Galkina, 2012; Leitinger and Schulman, 2013). It must be noted that also smooth muscle cells (SMCs) can assume some of the functions usually assigned to $\mathrm{M} \Phi$ s (efferocytosis, internalization of lipids or cholesterols), and may transform in foam cells (Chistiakov et al., 2017).

In any case, cells with phenotypes closer to Mox or M1 cells are the most active in internalizing lipids (particularly cholesterol), especially if agglomerated within oxidized low density lipoprotein (oxLDL). Upon this process, efferocytosis efficiency of cells is reduced, they transform into foam cells, and are easily subjected to imbalances in cholesterol influx/efflux process and hypoxia. The final outcome is most often apoptosis or necrosis, which resolve in local accumulation of the lipid content, the major constituent of the inner (possibly necrotic) core of the atherosclerotic plaque (Moore and Tabas, 2011). At the same time, the cell's residual components promote further inflammation signals and generation of oxidative species, in a persistent cycle of recruitment of intimal macrophages and their polarization toward pro-inflammatory subsets (Libby et al., 2014). Moreover, both monocytes and macrophages have been shown to contribute to the increase of the gamma-glutamyl transferase enzyme $(\gamma \mathrm{GT})$ in AS lesions, increasing the oxidative character of these zones (Pucci et al., 2014; Belcastro et al., 2015).

This self-sustained state of inflammation (similar to what happens in chronic wound environments) is characteristic of the vulnerable plaque. The presence of activated macrophages leads to the secretion of matrix-components degrading enzymes (such as matrix metalloproteinases-MMPs), inhibits the production of collagen by the SMCs, and finally contributes to vascular wall reshaping. These mechanisms are useful in early lesions, supporting resolution of disrupted endothelial layers and avoiding accumulation of toxic species, as well as for the reduction of atherosclerotic plaques; but, at the same time, they induce fragility in late plaques, with the possible final consequence of plaque rupture (Libby et al., 2014; Hansson et al., 2015; Martinez and White, 2018). Inflammation also modulates the clinical consequences of the thrombotic complications of AS, while its inhibition could attenuate progression, mitigate the risk of plaque rupture, and even promote regression of AS (Awan and Genest, 2015; Bäck and Hansson, 2015; Bäck et al., 2015; Kamaly et al., 2016).

\section{ATHEROSCLEROSIS TREATMENTS}

Current therapeutic approaches to AS aim at reducing promoting factors, including hypertension, smoke, and dyslipidemias; considered drugs (e.g., inhibitors of cholesterol hepatic synthesis like statins) particularly affect production and transport of cholesterol (or other lipids) to the arterial walls (Coomes et al., 2011; Duivenvoorden et al., 2014; Tsujita et al., 2015). Different approaches have been investigated, such as blocking absorption of cholesterol in the intestine (e.g., by ezetamibe) or controlling its reverse transport in ICs, e.g., by using an agonist for the liver X receptor (LXR) like GW3965, or administering artificial forms of high density lipoproteins (HDL; Duivenvoorden et al., 2014; Tsujita et al., 2015; Pulakazhi Venu et al., 2017; Goldberg, 2018; Mueller et al., 2018).

Controlling inflammation is another promising strategy; in particular, the interleukin-1 pathway has been identified as a possible therapeutic target. Canakinumab, a monoclonal antibody targeting interleukin- $1 \beta$, was tested in the CANTOS (Canakinumab ANti-inflammatory Thrombosis Outcomes Study) trial (Ridker et al., 2011, 2017). Moreover, the chemotherapeutic drug methotrexate (MTX), largely used as an immunomodulatory and anti-inflammatory (AI) drug, was described to lower the risk for total cardiovascular diseases in patients with chronic inflammation (Popkova et al., 2015; Gomes et al., 2018), and has been observed to inhibit atherogenesis and macrophage migration to the intima in animal models (Bulgarelli et al., 2012). Although its mechanisms of action are not fully clear, this molecule is capable of reducing the secretion of pro-inflammatory cytokines and the expression of adhesion molecules on both immune and endothelial cells (Coomes et al., 2011).

While promising, systemic administration of AI drugs is often limited by a narrow therapeutic index and by severe adverse effects, including bone marrow suppression, neutropenia and immunodepression. Blockade and stimulation of the mechanisms involved in inflammation can hold positive, null, or even detrimental results depending on the phase of the atherogenic process (Aluganti Narasimhulu et al., 2016). Based 
on these premises, it is authors' belief that a rational modulation of the functions of ICs is promising for an efficient treatment and hopefully comes with fewer adverse effects.

Ideally, it would be desirable to identify an effective strategy for all of the AS phases; e.g., in (Libby et al., 2014) Resolvin E1 is indicated as a mediator that reverses all the advanced lesions associated processes, while contributing to the resolution of the plaque also in earlier phases. Another promising possibility is controlling release or activation of compounds used to alleviate or cure AS symptoms/consequences. Possible approaches include: the use of drugs modified with a glutamyl in order to exploit the increased concentration of $\gamma \mathrm{GT}$ in AS lesions (Belcastro et al., 2015); developing methods exploiting high cholesterol concentrations; tackling pathways leading the transformation process from monocyte to pro-inflammatory MФs and finally to foam cells (Rousselle et al., 2013).

Due to their ambivalent role of inducing inflammation and regulating tissue regeneration, macrophages have been the first candidates addressed in order to control AS. Vannella and Wynn presented an interesting review describing macrophages behavior in different tissues, the mediators of the various mechanisms involved, and finally several possible ways to modulate them (Vannella and Wynn, 2017). It would be ideal to force their egress from AS lesions as transformation into foam cells is occurring; to this end, a suggestive idea could be activating or releasing a "macrophage migration-enhancement factor" (Weisbart et al., 1974; Ueno et al., 1997; Nunami et al., 1998), or interfering with cell surface adhesion molecule only in overabundancy of cholesterol or other AS markers, e.g. by stopping the phosphorylation of CD44 (Qin, 2012).

\section{TOWARD TARGETED THERANOSTICS NPS}

As hinted above, spatial and temporal control of drug activity can reduce collateral short and long term effects; moreover, it can result in more convenient administration methods. These premises strongly call for the development of efficient, targeted delivery strategies for AI molecules, just like the ones based on their encapsulation into NPs (Costa Lima and Reis, 2015). Several kinds of NP have been considered for drug delivery; characteristics and production protocols for some of these are reviewed in Allen et al. (2016), Ulbrich et al. (2016), Cheraghi et al. (2017), and Matoba et al. (2017). There are still limitations and drawbacks for the clinical use of many NPs; these could arise from a not-yet perfect control of the final fate of many formulations, since they often accumulate also in the organs of the reticuloendothelial system (RES), from polydispersion and/or poor reproducibility in their preparation, or from the often difficult scale-up and high cost for their production, especially when multifunctional capabilities are added (Cheng et al., 2012; Ulbrich et al., 2016). However, NP physicochemical properties can be finely tailored during their synthesis and this allows optimizing drug loading and NP target specificity (Allen et al., 2016; Pentecost et al., 2016).

In the clinical setting of atherosclerotic disease, NPs loaded with anti-inflammatory drugs can be a powerful tool to hit inflammatory targets at the plaque level, preventing systemic side effects (Jokerst and Gambhir, 2011; Di Mascolo et al., 2013). Drug-loaded NPs targeting macrophages and other immune cells could control their pro-inflammatory activities and thus prevent, attenuate, and possibly reverse related disorders (i.e., increased atherosclerosis, but also altered adipocyte function and insulin resistance). At the same time, NPs could also be loaded with imaging agents allowing the detection of vulnerable atherosclerotic plaques; similar theranostic strategies already showed potential for detection and treatment of other diseases (including cancer and neurodegenerative disorders), exploiting a number of imaging modalities, among which optical imaging (OI), magnetic resonance imaging (MRI), ultrasound and photoacoustic (US-PA), computed tomography (CT), and nuclear imaging based on single photon and positron emission tomography (SPECT, PET; Xie et al., 2010; Kim et al., 2014; Weissleder et al., 2014; Atukorale et al., 2017; Stigliano et al., 2017; Zhang et al., 2017).

There is evidence that NPs can segregate into the plaque in preclinical models of AS via a "passive" targeting mechanism (Weissleder et al., 2014); while such a relative selectivity has not been completely understood, some hypotheses have been proposed. First, the abnormal hemodynamic forces where plaques develop may favor NPs deposition (Hossain et al., 2015); second, the endothelium appears discontinuous with openings for sufficiently small objects (Kim et al., 2014); third, there can be an active role of immune cells in vehiculating or accumulating the nanoparticles in inflamed districts (Moore et al., 2017).

Other strategies focused on specific targeting, e.g., toward inflammatory factors, dysfunctional endothelial cells, or specific macrophage receptors involved in cholesterol transport. Examples are drug carriers functionalization with selectin ligands or antibodies directed to CAMs (sialyl-Lewis X, PSFL-1, ICAM and VICAM ligands, anti-ICAM, anti-VCAM; Robbins et al., 2010) or anti-oxLDL receptor (Li et al., 2010). Li et al. used liposomes decorated with LOX-1 antibodies, Indium $\left({ }^{111} \mathrm{In}\right)$ or Gadolinium (Gd), and DiI fluorescence markers to image atherosclerotic plaques in $\mathrm{ApoE}^{-/-}$mice. Alternatively, it has been proposed to target collagen IV, which is present on the vascular basement and exposed when vascular permeability increases (Chan et al., 2011; Chen et al., 2013; Kamaly et al., 2016; Meyers et al., 2017). Also common strategies to target macrophages are based on functionalizing nanoparticles with dextran sulfate coating or peptides mimicking low-density lipoproteins (LDL) such as apolipoprotein A1 (ApoA-1), whose receptors (Class A Scavenger Receptor 1 MSR-1, and class B Scavenge Receptor CD36) are expressed on macrophages cell membrane (Canton et al., 2013). Further works focusing on visualization of macrophages distribution using nanoparticles are well reviewed in Weissleder et al. (2014).

Active targeting was achieved also using a "biomimetic" approach: NPs can derive from, or have properties similar to, aggregates like LDL or HDL, naturally accumulating in AS plaques (Allijn et al., 2013; Duivenvoorden et al., 2014; Gomes et al., 2018). More specific tissue or cell targeting can be found testing nanoparticle libraries in-vitro or in-vivo (Kamaly et al., 2016; Tang et al., 2016), but the mechanisms of the found 
specificity should be understood, also in order to ensure it is preserved downhill of modifications of nanoconstructs or in different biological environments. Libraries are often tested preliminarily in cell cultures, but in-vivo tests are necessary at least to evaluate the impact of the different biological media.

Indeed, upon entry in an organism, NPs are usually coated by a protein corona (PC), changing their biological identity. PC formation (or "opsonisation") is often the first step toward the sequestration of NPs by the RES. Various approaches have been considered to avoid such phenomenon. Recent strategies are based on controlling nanoconstruct stiffness, since it was found that deformable particles are less subject to uptake by macrophages in RES in off-target tissues (Key et al., 2015; Palomba et al., 2018). Other methods exploit different coatings and functionalization developed for controlling the PC, e.g., by using polymers like PEG. This is thought to be an antifouling agent, but it actually seems to modulate the PC (Schöttler et al., 2016); moreover, it has been shown to be immunogenic, requiring the use of additional functionalizing moieties (Mima et al., 2017). Other possible coating molecules are based on peptides: examples comprise zwitterionic ones, for limiting serum-protein adsorption (Ranalli et al., 2017), or aptamer-likes, for enriching the PC with specific molecules present in biological fluids, which act as targeting moieties when properly oriented (Santi et al., 2017). All these approaches easily grant nanoconstructs with extended circulation half-life.

An advantage that will play important role consists in NPs potential multimodality: not only they can contain more than one drug and/or imaging agent, but it could also be possible to implement a trigger for drug release/activation. This could either be intrinsic (provided by the pathological environment) or exogenous (ultrasound, light, oscillating magnetic fields).

Especially under this view, an interesting development can arise from a synergy between nanotechnology and personalized medicine (Mendes et al., 2018). Early screening of the most suited bioactives (Tang et al., 2016; Risum et al., 2017), real time monitoring of local accumulations (Zavaleta et al., 2018), observing early feedbacks to the treatment (Qiao et al., 2017), and predicting patient responses (Sykes et al., 2016) are all possible aspects of personalized medicine. We believe that the rational application of nanotools in all these steps will embody a fundamental role in the upcoming clinical and pre-clinical research (Mura and Couvreur, 2012; Polyak and Ross, 2017; Yordanova et al., 2017).

\section{NPs FOR AS TREATMENT AND DIAGNOSIS}

The use of NPs for treatment of AS and visualization of plaques up to 2015 has been elegantly reviewed in Zhang et al. (2017); we further selected more recent works not reported there (Table 1). In this session, we describe some of these and other relevant works.

Often, intrinsic properties of NPs lead to the exploitation of drug delivery strategies together with photothermal- and radiofrequency-mediated triggering effects; e.g., Johnston proposed the possibility to use photothermal destruction of macrophages using iron oxide NPs with thin gold and dextran coating, excited by a laser pulse at $755 \mathrm{~nm}$. In this manuscript the particles were used for MRI and in vitro photothermolysis (Ma et al., 2009). A similar approach has been tested in the NANOMFIM trial (Kharlamov et al., 2015); here, NPs composed by silica shells containing gold and eventually magnetic nanoparticles were delivered on AS plaques by a bioengineered on-artery patch or using a magnetic navigation system; detonation of NPs using a NIR laser caused a significant final reduction of the total atheroma volume.

A different, interesting strategy consists on preventively acting on the selective recruitment of monocytes from the precursors of pro-inflammatory macrophages M1 (Nakashiro et al., 2016; Matoba et al., 2017). The authors proposed polymeric NPs loaded with Pioglitazone, an agonist of the receptor $\operatorname{PPAR} \gamma$ shown to be able to influence macrophage polarization. The formulation was tested in $\mathrm{ApoE}^{-/-}$mice fed a high fat diet (HFD) and infused with angiotensin II, promoting inflammation driven by monocytes/macrophages. 2 days post injection the ratio between peripheral pro- and non-inflammatory monocytes subsets decreased substantially, and tissue macrophage polarity was regulated toward the noninflammatory phenotype M2, with consequent suppression of EMMPRIN/MMP pathway and reduction of plaque destabilization risk.

In a similar approach, Stigliano et al. (2017) confirmed the potentiality of MTX in preventive-oriented treatments by loading it into NPs. The authors demonstrated specific accumulation of NPs into macrophages residing within lipid-rich plaques along the arterial tree. In the aortic arch of $\mathrm{ApoE}^{-/-}$mice on HFD treated with MTX-NPs, 50\% less coverage of plaques was found in comparison to the control group. Importantly, this result was obtained by injecting a dose four times lower than reported in literature (Leite et al., 2015; Gomes et al., 2018).

MTX has also been investigated in synergy with different bioactive compounds. The group of Serrano Jr. (Gomes et al., 2018) tested the combined effect of injecting Paclitaxel (PTX)loaded LDL-mimicking NPs and MTX-loaded NPs. In New Zealand white rabbits on atherogenic diet treated with both the NPs formulations, the regression of the lesion area and the intimal width reduction corresponded to 17 and $63 \%$, respectively, compared to the group treated with PTX-NPs alone. The authors speculate that this athero-regression is mostly due to the macrophage reduction effect and not to an inhibition of SMCs migration into the intima.

Other possible applications of nanomedicine in the treatment of AS are based on gene regulation. Majmudar et al injected siRNA-containing polymeric NPs to silence the expression of $\mathrm{C}-\mathrm{C}$ chemokine receptor type 2 (CCR-2), a key player in recruiting inflamed monocytes to atherosclerotic plaques; they observed reduced PET signals from ${ }^{89} \mathrm{Zr}$-labeled dextran nanoparticles in aortic root when compared to mice treated with an irrelevant siRNA (Majmudar et al., 2013). Another example is the downregulation of the tissue inhibitor of metalloproteinase 3 by the use of miR-712, delivered by cationic lipid NPs targeting VCAM1. The treatment was performed in $\mathrm{ApoE}^{-/-}$ 


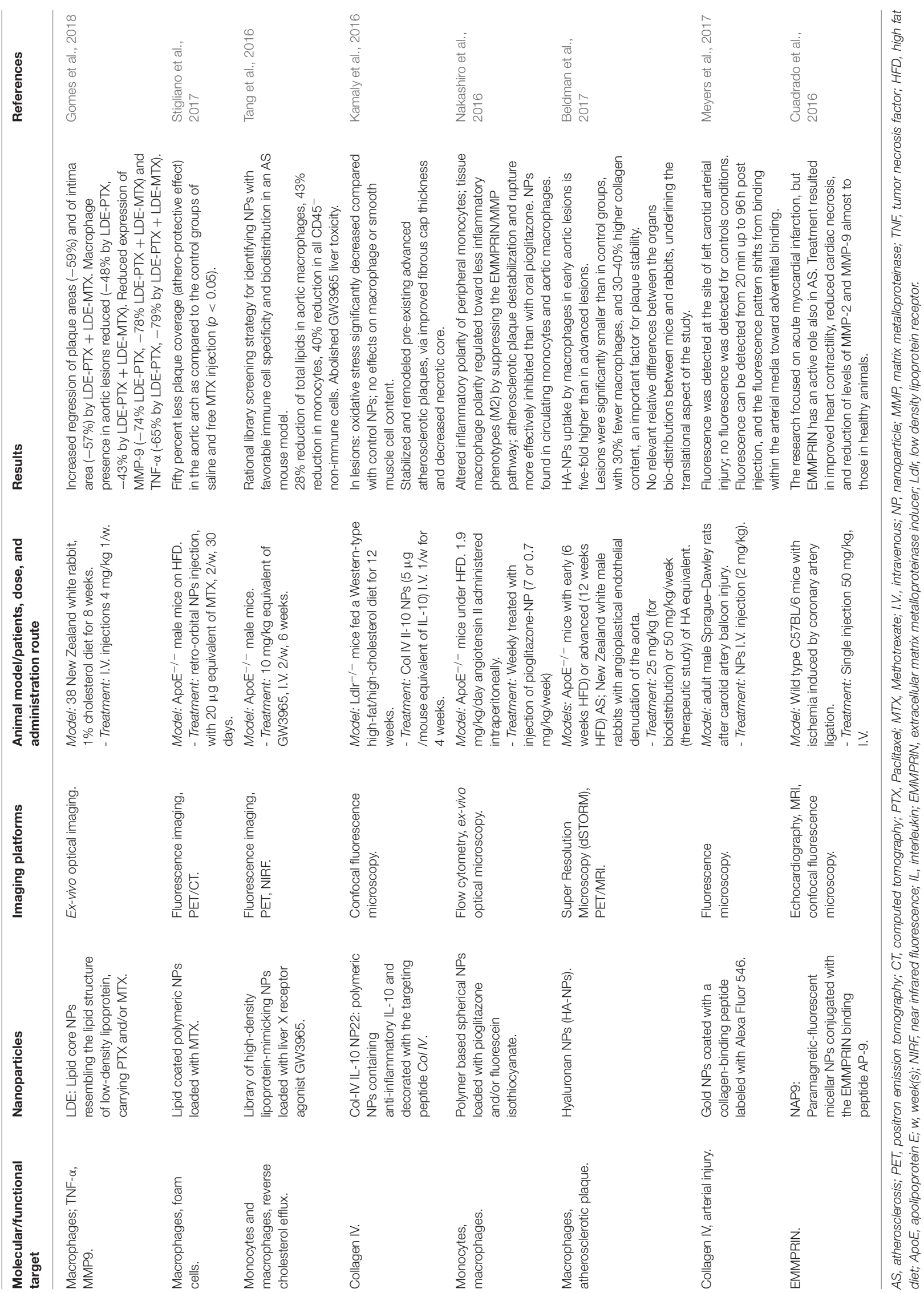




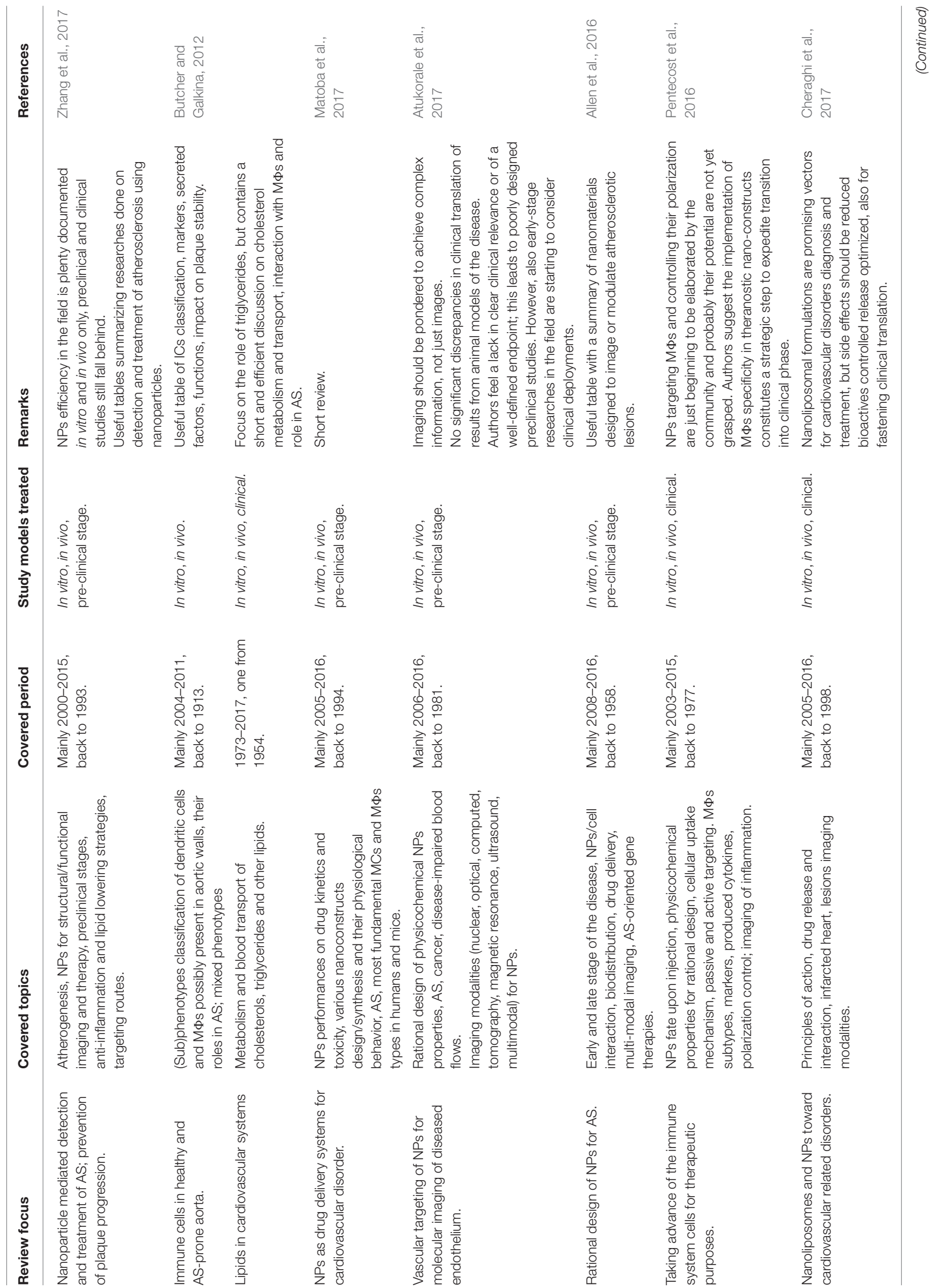




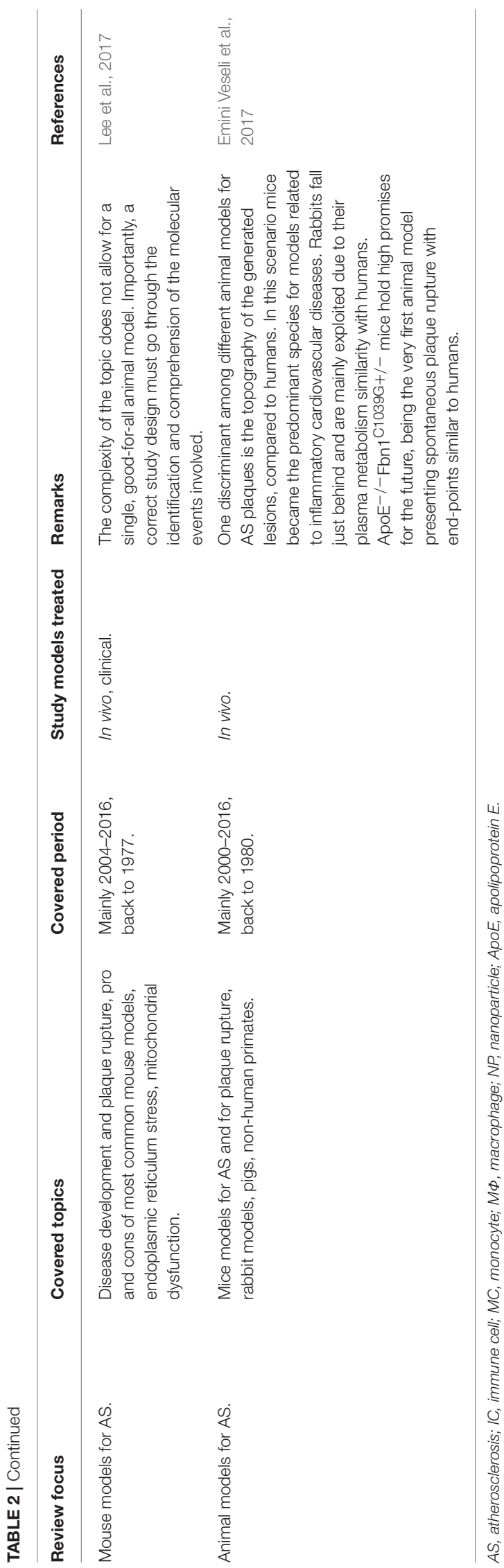

mice and was able to significantly attenuate the development of atherosclerotic plaques (Kheirolomoom et al., 2015).

\section{TOOLS AND IDEAS FOR ADDITIONAL RESEARCH}

As reviewed above, fundamental and pre-clinical researches on AI-drugs-loaded NPs against AS are beginning to bloom, and there are also clinical trials for the (separated) use of NPs and AI drugs for regression or consolidation of AS plaques. More fundamental and translational research on the mechanisms underlying their action could inspire and motivate future more efficient clinical trials using AI-drug loaded NPs against AS.

Important future steps for the development of new NP-based theranostics strategies can arise from the study of interaction mechanisms between NPs and immune cells, with particular regard to what fate NPs and their components will face upon site deposition and cellular uptake. Investigations on these directions will remarkably improve the development of NPs rational designs aimed at specific accumulation and cargo smart activation.

Indeed, where, how and in which proportion NPs are internalized in the various subtypes of ICs is still not clear, also due to the different internalization routes observed even in close phenotypes (Lunov et al., 2011), nor it is clear how or if they are exocytosed (Oh and Park, 2014). In particular, are NPs mostly internalized by circulating monocytes that enter the lesions, or by resident macrophages in lesions, where NPs enter because of the enhanced permeability of inflamed endothelium?

Immortalized cell lines can be used for preliminary experiments. The most used are murine macrophage-like RAW 264.7 and J774, and human monocyte-like THP-1 and U937 (Luster et al., 1995; Qin, 2012; Andreu et al., 2017) reviews in particular the use of THP-1, unchanged or differentiated toward a $M \Phi$ phenotype (especially $\mathrm{M} 1$ ), but also cites other human monocyte/macrophage models. Following these models, even though there are evident parallelisms, care must be taken for intrinsic differences: between cells originating from different organisms (Ingersoll et al., 2010; Matoba et al., 2017; Zhang et al., 2017); between immortalized cell lines and primary cells (Andreu et al., 2017); even between possible different differentiations ICs may undergo during regular cultures. Primary cells should be used in final tests, but in this case the last type of unwanted differentiations are even more critical [e.g., monocytes are extremely sensible and may be activated just by sole adhering on surfaces (Belcastro et al., 2015)]. For these reasons, a thoughtful characterization of their phenotypes (by visual inspection or, better, by markers analysis) should be carried out before every experiment.

Together with the internalization pathway, also the biodistribution of NPs deserves attention; a nice review for both these issues, which considers $M C$ and $M \Phi s$, can be found in Pentecost et al. (2016), with interesting discussions about dependencies on NP geometry and surface chemistry, targeting, control on $M \Phi$ phenotype, and imaging. In addition, medium and long-term fates of nano-construct components (in particular its bioactive cargo) should be addressed; these details can indeed 
impact the efficacy of the researched treatment, but also the long-term effects on the patients' health.

Finally, various animal models have been developed for the different phases of AS, as reviewed in Emini Veseli et al. (2017) and Lee et al. (2017). A recently developed model of controllable and reversible hypercholesterolemia is based on transient knockdown of the hepatic LDL receptor (LDLR) by antisense oligonucleotides in wild type C57BL/6 mice, followed by its rapid restoration (Basu et al., 2018).

\section{CONCLUSION}

Cardiovascular events, such as acute myocardial infarction and stroke, are often associated with erosion/rupture of arterial atherosclerotic plaques and superimposed thrombosis. These can cause arterial-vessel occlusion, downstream ischemia and necrosis, with subsequent heart failure and the whole clinical spectrum of vascular encephalopathies. Being AS an important source of morbidity and mortality, intense research efforts toward precision and personalized medicine in this field are motivated.

There are several reviews for selected aspects of the highly complicated issue of AS and its theranostics with NPs (Table 2). Here, we shortly presented the broad background necessary to understand the use of NPs for smart delivery and activation of anti-inflammatory drugs. We tried to focus on how basic science, together with the translational and clinical aspects, can address different portions of this, aiming at providing a better

\section{REFERENCES}

Allen, S., Liu, Y. G., and Scott, E. (2016). Engineering nanomaterials to address cellmediated inflammation in atherosclerosis. Regen. Eng. Transl. Med. 2, 37-50. doi: 10.1007/s40883-016-0012-9

Allijn, I. E., Leong, W., Tang, J., Gianella, A., Mieszawska, A. J., Fay, F., et al. (2013). Gold nanocrystal labeling allows low-density lipoprotein imaging from the subcellular to macroscopic level. ACS Nano 7, 9761-9770. doi: $10.1021 / \mathrm{nn} 403258 \mathrm{w}$

Aluganti Narasimhulu, C., Fernandez-Ruiz, I., Selvarajan, K., Jiang, X., Sengupta, B., Riad, A., et al. (2016). Atherosclerosis - do we know enough already to prevent it? Curr. Opin. Pharmacol. 27, 92-102. doi: 10.1016/j.coph.2016.02.006

Andreu, N., Phelan, J., De Sessions, P. F., Cliff, J. M., Clark, T. G., and Hibberd, M. L. (2017). Primary macrophages and J774 cells respond differently to infection with Mycobacterium tuberculosis. Sci. Rep. 7:42225. doi: 10.1038/srep42225

Atukorale, P. U., Covarrubias, G., Bauer, L., and Karathanasis, E. (2017). Vascular targeting of nanoparticles for molecular imaging of diseased endothelium. $A d v$. Drug Deliv. Rev. 113, 141-156. doi: 10.1016/j.addr.2016.09.006

Awan, Z., and Genest, J. (2015). Inflammation modulation and cardiovascular disease prevention. Eur. J. Prev. Cardiol. 22, 719-733. doi: $10.1177 / 2047487314529350$

Bäck, M., and Hansson, G. K. (2015). Anti-inflammatory therapies for atherosclerosis. Nat. Rev. Cardiol. 12:199. doi: 10.1038/nrcardio.2015.5

Bäck, M., Weber, C., and Lutgens, E. (2015). Regulation of atherosclerotic plaque inflammation. J. Intern. Med. 278, 462-482. doi: 10.1111/joim.12367

Basu, D., Hu, Y., Huggins, L.-A., Mullick, A. E., Graham, M. J., Wietecha, T., et al. (2018). Novel reversible model of atherosclerosis and regression using oligonucleotide regulation of the LDL receptor. Circ. Res. 122, 560-567. doi: 10.1161/CIRCRESAHA.117.311361

Belcastro, E., Franzini, M., Cianchetti, S., Lorenzini, E., Masotti, S., Fierabracci, V., et al. (2015). Monocytes/macrophages activation answer for the underlying mechanisms of NPs intra-plaque internalization, as well as for the molecular and cellular processes at the bases of different treatments. We stressed that a more thorough understanding of these would help foresee possible side effects, even long term ones, and also the opportunity to employ similar techniques and (nano)tools to other pathologies.

While single studies can be carried out in specialized groups, a deeper understanding of concepts, mechanisms and applicability of the developed (nano)tools needs a highly interdisciplinary environment, involving e.g. cardiologists, biologists, physicists, chemists, engineers; professionals able to talk with each other, and fostering the formation of multi-faceted scientists.

\section{AUTHOR CONTRIBUTIONS}

SL conceived the idea. SL and AC wrote the manuscript, with major contributions from RP, RC, and GV, starting from texts written in collaboration with LM, ME, and PD. All authors discussed, edited, and contributed to the manuscript.

\section{FUNDING}

SL acknowledges funding from Scuola Normale Superiore (university coordinated project Visualization and theranostic of atherosclerotic PLAQUEs VIA compound NANOparticles, PLAQUEviaNANO, in collaboration with Scuola Superiore Sant'Anna) and Fondazione Pisa (project Nanotechnology for tumor molecular fingerprinting and early diagnosis, RST 148/16).

contributes to b-gamma-glutamyltransferase accumulation inside atherosclerotic plaques. J. Transl. Med. 13:325. doi: 10.1186/s12967-0150687-6

Beldman, T. J., Senders, M. L., Alaarg, A., PéRez-Medina, C., Tang, J., Zhao, Y., et al. (2017). Hyaluronan nanoparticles selectively target plaque-associated macrophages and improve plaque stability in atherosclerosis. ACS Nano 11, 5785-5799. doi: 10.1021/acsnano.7b01385

Bulgarelli, A., Dias, A. A. M., Caramelli, B., and Maranhão, R. C. (2012). Treatment with methotrexate inhibits atherogenesis in cholesterol-fed rabbits. J. Cardiovasc. Pharmacol. 59, 308-314. doi: 10.1097/FJC.0b013e318241c385

Butcher, M. J., and Galkina, E. V. (2012). Phenotypic and functional heterogeneity of macrophages and dendritic cell subsets in the healthy and atherosclerosisprone aorta. Front. Physiol. 3:44. doi: 10.3389/fphys.2012.00044

Canton, J., Neculai, D., and Grinstein, S. (2013). Scavenger receptors in homeostasis and immunity. Nat. Rev. Immunol. 13, 621-634. doi: $10.1038 /$ nri3515

Chan, J. M., Rhee, J.-W., Drum, C. L., Bronson, R. T., Golomb, G., Langer, R., et al. (2011). In vivo prevention of arterial restenosis with paclitaxel-encapsulated targeted lipid-polymeric nanoparticles. Proc. Natl. Acad. Sci. 108, 19347-19352. doi: 10.1073/pnas.1115945108

Chen, W., Cormode, D. P., Vengrenyuk, Y., Herranz, B., Feig, J. E., Klink, A., et al. (2013). Collagen-specific peptide conjugated HDL nanoparticles as MRI contrast agent to evaluate compositional changes in atherosclerotic plaque regression. JACC Cardiovasc. Imaging 6, 373-384. doi: 10.1016/j.jcmg.2012.06.016

Cheng, Z., Al Zaki, A., Hui, J. Z., Muzykantov, V. R., and Tsourkas, A. (2012). Multifunctional nanoparticles: cost versus benefit of adding targeting and imaging capabilities. Science 338, 903-910. doi: 10.1126/science.1226338

Cheraghi, M., Negahdari, B., Daraee, H., and Eatemadi, A. (2017). Heart targeted nanoliposomal/nanoparticles drug delivery: an updated review. Biomed. Pharmacother. 86, 316-323. doi: 10.1016/j.biopha.2016.12.009 
Chistiakov, D. A., Myasoedova, V. A., Revin, V. V., Orekhov, A. N., and Bobryshev, Y. V. (2017). The phenomenon of atherosclerosis reversal and regression: Lessons from animal models. Exp. Mol. Pathol. 102, 138-145. doi: 10.1016/j.yexmp.2017.01.013

Coomes, E., Chan, E. S., and Reiss, A. B. (2011). Methotrexate in atherogenesis and cholesterol metabolism. Cholesterol 2011:503028. doi: 10.1155/2011/503028

Costa Lima, S. A., and Reis, S. (2015). Temperature-responsive polymeric nanospheres containing methotrexate and gold nanoparticles: a multi-drug system for theranostic in rheumatoid arthritis. Colloids Surfaces B 133, 378-387. doi: 10.1016/j.colsurfb.2015.04.048

Cuadrado, I., Piedras, M. J. G. M., Herruzo, I., Del Carmen Turpin, M., Castejón, B., Reventun, P., et al. (2016). EMMPRIN-targeted magnetic nanoparticles for in vivo visualization and regression of acute myocardial infarction. Theranostics 6:545. doi: 10.7150/thno.13352

Di Mascolo, D., Lyon, C. J., Aryal, S., Ramirez, M. R., Wang, J., Candeloro, P., et al. (2013). Rosiglitazone-loaded nanospheres for modulating macrophage-specific inflammation in obesity. J. Controll. Release 170, 460-468. doi: 10.1016/j.jconrel.2013.06.012

Duivenvoorden, R., Tang, J., Cormode, D. P., Mieszawska, A. J., IzquierdoGarcia, D., Ozcan, C., et al. (2014). A statin-loaded reconstituted high-density lipoprotein nanoparticle inhibits atherosclerotic plaque inflammation. Nat. Commun. 5:3065. doi: 10.1038/ncomms4065

Emini Veseli, B., Perrotta, P., De Meyer, G. R. A., Roth, L., Van Der Donckt, C., Martinet, W., et al. (2017). Animal models of atherosclerosis. Eur. J. Pharmacol. 816, 3-13. doi: 10.1016/j.ejphar.2017.05.010

Fisher, E. A. (2016). The regression of atherosclerosis-the journey from the liver to the plaque and back. Arterioscler. Thromb. Vasc. Biol. 36:226. doi: 10.1161/ATVBAHA.115.301926

Goldberg, I. J. (2018). Fat in the blood, fat in the artery, fat in the heart: triglyceride in physiology and disease. Arterioscler. Thrombosis Vasc. Biol. 38, 700-706. doi: 10.1161/ATVBAHA.117.309666

Gomes, F. L., Maranhão, R. C., Tavares, E. R., Carvalho, P. O., Higuchi, M. L., Mattos, F. R., et al. (2018). Regression of atherosclerotic plaques of cholesterolfed rabbits by combined chemotherapy with paclitaxel and methotrexate carried in lipid core nanoparticles. J. Cardiovasc. Pharmacol. Ther. 2018:8836. doi: $10.1177 / 1074248418778836$

Hansson, G. K., Libby, P., and Tabas, I. (2015). Inflammation and plaque vulnerability. J. Intern. Med. 278, 483-493. doi: 10.1111/joim.12406

Hossain, S. S., Zhang, Y., Fu, X., Brunner, G., Singh, J., Hughes, T. J., et al. (2015). Magnetic resonance imaging-based computational modelling of blood flow and nanomedicine deposition in patients with peripheral arterial disease. J. R. Soc. Interface 12:20150001. doi: 10.1098/rsif.2015.0001

Ingersoll, M. A., Spanbroek, R., Lottaz, C., Gautier, E. L., Frankenberger, M., Hoffmann, R., et al. (2010). Comparison of gene expression profiles between human and mouse monocyte subsets. Blood 115, e10-e19. doi: 10.1182/blood-2009-07-235028

Jokerst, J. V., and Gambhir, S. S. (2011). Molecular imaging with theranostic nanoparticles. Acc. Chem. Res. 44, 1050-1060. doi: 10.1021/ar200106e

Kamaly, N., Fredman, G., Fojas, J. J., Subramanian, M., Choi, W. I., Zepeda, K., et al. (2016). Targeted interleukin-10 nanotherapeutics developed with a microfluidic chip enhance resolution of inflammation in advanced atherosclerosis. ACS Nano 10, 5280-5292. doi: 10.1021/acsnano. 6b01114

Key, J., Palange, A. L., Gentile, F., Aryal, S., Stigliano, C., Di Mascolo, D., et al. (2015). Soft discoidal polymeric nanoconstructs resist macrophage uptake and enhance vascular targeting in tumors. ACS Nano 9, 11628-11641. doi: $10.1021 /$ acsnano.5b04866

Kharlamov, A. N., Tyurnina, A. E., Veselova, V. S., Kovtun, O. P., Shur, V. Y., and Gabinsky, J. L. (2015). Silica-gold nanoparticles for atheroprotective management of plaques: results of the NANOM-FIM trial. Nanoscale 7, 8003-8015. doi: 10.1039/C5NR01050K

Kheirolomoom, A., Kim, C. W., Seo, J. W., Kumar, S., Son, D. J., Gagnon, M. K. J., et al. (2015). Multifunctional nanoparticles facilitate molecular targeting and miRNA delivery to inhibit atherosclerosis in ApoE-/-mice. ACS Nano 9, 8885-8897. doi: 10.1021/acsnano.5b02611

Kim, Y., Lobatto, M. E., Kawahara, T., Chung, B. L., Mieszawska, A. J., SanchezGaytan, B. L., et al. (2014). Probing nanoparticle translocation across the permeable endothelium in experimental atherosclerosis. Proc. Natl. Acad. Sci. 111, 1078-1083. doi: 10.1073/pnas.1322725111

Lee, Y. T., Lin, H. Y., Chan, Y. W. F., Li, K. H. C., To, O. T. L., Yan, B. P., et al. (2017). Mouse models of atherosclerosis: a historical perspective and recent advances. Lipids Health Dis. 16:12. doi: 10.1186/s12944-016-0402-5

Leite, A. C., Solano, T. V., Tavares, E. R., and Maranhão, R. C. (2015). Use of combined chemotherapy with etoposide and methotrexate, both associated to lipid nanoemulsions for atherosclerosis treatment in cholesterol-fed rabbits. Cardiovasc. Drugs Ther. 29, 15-22. doi: 10.1007/s10557-014-6566-1

Leitinger, N., and Schulman, I. G. (2013). Phenotypic polarization of macrophages in atherosclerosis. Arterioscler. Thromb. Vasc. Biol. 33, 1120-1126. doi: 10.1161/ATVBAHA.112.300173

Li, D., Patel, A. R., Klibanov, A. L., Kramer, C. M., Ruiz, M., Kang, B.-Y., et al. (2010). Molecular imaging of atherosclerotic plaques targeted to oxidized LDL receptor LOX-1 by SPECT/CT and magnetic resonance. Circulation 3, 464-472. doi: 10.1161/CIRCIMAGING.109.896654

Libby, P., Tabas, I., Fredman, G., and Fisher, E. A. (2014). Inflammation and its resolution as determinants of acute coronary syndromes. Circ. Res. 114, 1867-1879. doi: 10.1161/CIRCRESAHA.114.302699

Lunov, O., Syrovets, T., Loos, C., Beil, J., Delacher, M., Tron, K., et al. (2011). Differential uptake of functionalized polystyrene nanoparticles by human macrophages and a monocytic cell line. ACS Nano 5, 1657-1669. doi: $10.1021 / \mathrm{nn} 2000756$

Luster, A. D., Greenberg, S. M., and Leder, P. (1995). The IP-10 chemokine binds to a specific cell surface heparan sulfate site shared with platelet factor 4 and inhibits endothelial cell proliferation. J. Exp. Med. 182, 219-231. doi: $10.1084 / \mathrm{jem} .182 .1 .219$

Ma, L. L., Feldman, M. D., Tam, J. M., Paranjape, A. S., Cheruku, K. K., Larson, T. A., et al. (2009). Small multifunctional nanoclusters (nanoroses) for targeted cellular imaging and therapy. ACS Nano 3, 2686-2696. doi: 10.1021/nn900440e Majmudar, M. D., Yoo, J., Keliher, E. J., Truelove, J. J., Iwamoto, Y., Sena, B., et al. (2013). Polymeric nanoparticle PET/MR imaging allows macrophage detection in atherosclerotic plaques. Circ. Res. 112, 755-761. doi: 10.1161/CIRCRESAHA.111.300576

Martinez, B. K., and White, C. M. (2018). The emerging role of inflammation in cardiovascular disease. Ann. Pharmacother. 52, 801-809. doi: $10.1177 / 1060028018765939$

Matoba, T., Koga, J.-I., Nakano, K., Egashira, K., and Tsutsui, H. (2017). Nanoparticle-mediated drug delivery system for atherosclerotic cardiovascular disease. J. Cardiol. 70, 206-211. doi: 10.1016/j.jjcc.2017.03.005

Mendes, M., Sousa, J., Pais, A., and Vitorino, C. (2018). "Clinical applications of nanostructured drug delivery systems: From basic research to translational medicine," in Core-Shell Nanostructures for Drug Delivery and Theranostics: Challenges, Strategies and Prospects for Novel Carrier Systems, In Woodhead Publishing Series in Biomaterials, eds M. L. Focarete and A. Tampieri (Cambridge, UK: Woodhead Publishing, Elsevier), 43-116. doi: 10.1016/B978-0-08-102198-9.00004-1

Meyers, M. W., Rink, J. S., Jiang, Q., Kelly, M. E., Vercammen, J. M., Thaxton, C. S., et al. (2017). Systemically administered collagen-targeted gold nanoparticles bind to arterial injury following vascular interventions. Physiol. Rep. 5:13128. doi: 10.14814 phy2.13128

Mima, Y., Abu Lila, A. S., Shimizu, T., Ukawa, M., Ando, H., Kurata, Y., et al. (2017). Ganglioside inserted into PEGylated liposome attenuates anti-PEG immunity. J. Controll. Release 250, 20-26. doi: 10.1016/j.jconrel.2017.01.040

Moore, K. J., Sheedy, F. J., and Fisher, E. A. (2013). Macrophages in atherosclerosis: a dynamic balance. Nat. Rev. Immunol. 13, 709-721. doi: 10.1038/nri3520

Moore, K. J., and Tabas, I. (2011). Macrophages in the pathogenesis of atherosclerosis. Cell 145, 341-355. doi: 10.1016/j.cell.2011.04.005

Moore, T. L., Hauser, D., Gruber, T., Rothen-Rutishauser, B., Lattuada, M., Petri-Fink, A., et al. (2017). Cellular shuttles: monocytes/macrophages exhibit transendothelial transport of nanoparticles under physiological flow. ACS Appl. Mater. Interfaces 9, 18501-18511. doi: 10.1021/acsami.7b03479

Mueller, P. A., Zhu, L., Tavori, H., Huynh, K., Giunzioni, I., Stafford, J. M., et al. (2018). Deletion of macrophage low-density lipoprotein receptor-related protein 1 (LRP1) accelerates atherosclerosis regression and increases CCR7 expression in plaque macrophages. Circulation 117:031702. doi: 10.1161/CIRCULATIONAHA.117.031702 
Mura, S., and Couvreur, P. (2012). Nanotheranostics for personalized medicine. Adv. Drug Deliv. Rev. 64, 1394-1416. doi: 10.1016/j.addr.2012. 06.006

Nakashiro, S., Matoba, T., Umezu, R., Koga, J.-I., Tokutome, M., Katsuki, S., et al. (2016). Pioglitazone-incorporated nanoparticles prevent plaque destabilization and rupture by regulating monocyte/macrophage differentiation in ApoE-/- mice. Arterioscler. Thromb. Vasc. Biol. 36, 491-500. doi: 10.1161/ATVBAHA.115.307057

Nunami, K.-I., Yamada, M., and Shimizu, R. (1998). Design of novel tripeptides with macrophage migration-enhancing activity. Bioorg. Med. Chem. Lett. 8, 2517-2520. doi: 10.1016/S0960-894X(98)00410-7

Oh, N., and Park, J.-H. (2014). Endocytosis and exocytosis of nanoparticles in mammalian cells. Int. J. Nanomedicine 9, 51-63. doi: 10.2147/IJN. S26592

Palomba, R., Palange, A. L., Rizzuti, I. F., Ferreira, M., Cervadoro, A., Barbato, M. G., et al. (2018). Modulating phagocytic cell sequestration by tailoring nanoconstruct softness. ACS Nano 12, 1433-1444. doi: 10.1021/acsnano.7b07797

Pentecost, A. E., Lurier, E. B., and Spiller, K. L. (2016). "Nanoparticulate systems for controlling monocyte/macrophage behavior," in Microscale Technologies for Cell Engineering, eds A. Singh and A. Gaharwar (Cham: Springer), 291-304.

Polyak, A., and Ross, T. L. (2017). Nanoparticles for SPECT and PET imaging: towards personalized medicine and theranostics. Curr. Med. Chem. 24, 1-26. doi: 10.2174/0929867324666170830095553

Popkova, T. V., Novikova, D. S., Gasparyan, A. Y., and Nasonov, E. L. (2015). Cardiovascular effects of methotrexate in rheumatoid arthritis revisited. Curr. Med. Chem. 22, 1903-1910. doi: 10.2174/0929867322666150415122039

Pucci, A., Franzini, M., Matteucci, M., Ceragioli, S., Marconi, M., Ferrari, M., et al. (2014). b-Gamma-glutamyltransferase activity in human vulnerable carotid plaques. Atherosclerosis 237, 307-313. doi: 10.1016/j.atherosclerosis.2014.09.028

Pulakazhi Venu, V. K., Adijiang, A., Seibert, T., Chen, Y.-X., Shi, C., Batulan, Z., et al. (2017). Heat shock protein 27-derived atheroprotection involves reverse cholesterol transport that is dependent on GM-CSF to maintain ABCA1 and ABCG1 expression in ApoE-/- mice. FASEB J. 31, 2364-2379. doi: 10.1096/fj.201601188R

Qiao, R., Qiao, H., Zhang, Y., Wang, Y., Chi, C., Tian, J., et al. (2017). Molecular imaging of vulnerable atherosclerotic plaques in vivo with osteopontin-specific upconversion nanoprobes. ACS Nano 11, 1816-1825. doi: 10.1021/acsnano.6b07842

Qin, Z. (2012). The use of THP-1 cells as a model for mimicking the function and regulation of monocytes and macrophages in the vasculature. Atherosclerosis 221, 2-11. doi: 10.1016/j.atherosclerosis.2011.09.003

Ranalli, A., Santi, M., Capriotti, L., Voliani, V., Porciani, D., Beltram, F., et al. (2017). Peptide-based stealth nanoparticles for targeted and $\mathrm{pH}$-triggered delivery. Bioconjug. Chem. 28, 627-635. doi: 10.1021/acs.bioconjchem.6b00701

Ridker, P. M., Everett, B. M., Thuren, T., Macfadyen, J. G., Chang, W. H., Ballantyne, C., et al. (2017). Antiinflammatory therapy with canakinumab for atherosclerotic disease. N. Engl. J. Med. 377, 1119-1131. doi: 10.1056/NEJMoa1707914

Ridker, P. M., Thuren, T., Zalewski, A., and Libby, P. (2011). Interleukin-1 $\beta$ inhibition and the prevention of recurrent cardiovascular events: rationale and design of the Canakinumab Anti-inflammatory Thrombosis Outcomes Study (CANTOS). Am. Heart J. 162, 597-605. doi: 10.1016/j.ahj.2011.06.012

Risum, S., Knigge, U., and Langer, S. W. (2017). Hitherto unseen survival in an ALK-positive-patient with advanced stage adult ganglioneuroblastoma treated with personalized medicine. Clin. Case Rep. 5, 2085-2087. doi: $10.1002 / \mathrm{ccr} 3.1262$

Robbins, G. P., Saunders, R. L., Haun, J. B., Rawson, J., Therien, M. J., and Hammer, D. A. (2010). Tunable leuko-polymersomes that adhere specifically to inflammatory markers. Langmuir 26, 14089-14096. doi: 10.1021/la1017032

Rousselle, A., Qadri, F., Leukel, L., Yilmaz, R., Fontaine, J.-F., Sihn, G., et al. (2013). CXCL5 limits macrophage foam cell formation in atherosclerosis. J. Clin. Invest. 123, 1343-1347. doi: 10.1172/JCI66580
Santi, M., Maccari, G., Mereghetti, P., Voliani, V., Rocchiccioli, S., Ucciferri, N., et al. (2017). Rational design of a transferrin-binding peptide sequence tailored to targeted nanoparticle internalization. Bioconjug. Chem. 28, 471-480. doi: 10.1021/acs.bioconjchem.6b00611

Schöttler, S., Becker, G., Winzen, S., Steinbach, T., Mohr, K., Landfester, K., et al. (2016). Protein adsorption is required for stealth effect of poly (ethylene glycol)and poly (phosphoester)-coated nanocarriers. Nat. Nanotechnol. 11:372. doi: 10.1038/nnano.2015.330

Stigliano, C., Ramirez, M. R., Singh, J. V., Aryal, S., Key, J., Blanco, E., et al. (2017). Methotraxate-loaded hybrid nanoconstructs target vascular lesions and inhibit atherosclerosis progression in ApoE(-/-) mice. Adv. Healthc. Mater. 6:201601286. doi: 10.1002/adhm.201601286

Sykes, E. A., Dai, Q., Sarsons, C. D., Chen, J., Rocheleau, J. V., Hwang, D. M., et al. (2016). Tailoring nanoparticle designs to target cancer based on tumor pathophysiology. Proc. Natl. Acad. Sci. 2016:201521265. doi: $10.1073 /$ pnas.1521265113

Taleb, S. (2016). Inflammation in atherosclerosis. Arch. Cardiovasc. Dis. 109, 708-715. doi: 10.1016/j.acvd.2016.04.002

Tang, J., Baxter, S., Menon, A., Alaarg, A., Sanchez-Gaytan, B. L., Fay, F., et al. (2016). Immune cell screening of a nanoparticle library improves atherosclerosis therapy. Proc. Natl. Acad. Sci. U.S.A. 113, E6731-E6740. doi: $10.1073 /$ pnas. 1609629113

Tsujita, K., Sugiyama, S., Sumida, H., Shimomura, H., Yamashita, T., Yamanaga, K., et al. (2015). Impact of dual lipid-lowering strategy with ezetimibe and atorvastatin on coronary plaque regression in patients with percutaneous coronary intervention: the multicenter randomized controlled PRECISE-IVUS trial. J. Am. Coll. Cardiol. 66, 495-507. doi: 10.1016/j.jacc.2015.05.065

Ueno, M., Sugita, T., Murakami, T., and Takata, I. (1997). The novel anti-rheumatic drug TA-383 has a macrophage migration enhancing activity. Jap. J. Pharmacol. 74, 221-224. doi: 10.1254/jjp.74.221

Ulbrich, K., Holá, K., Šubr, V., Bakandritsos, A., Tuček, J., and Zboril, R. (2016). Targeted drug delivery with polymers and magnetic nanoparticles: covalent and noncovalent approaches, release control, and clinical studies. Chem. Rev. 116, 5338-5431. doi: 10.1021/acs.chemrev.5b00589

Vannella, K. M., and Wynn, T. A. (2017). Mechanisms of organ injury and repair by macrophages. Annu. Rev. Physiol. 79, 593-617. doi: 10.1146/annurev-physiol-022516-034356

Weisbart, R. H., Bluestone, R., Goldberg, L. S., and Pearson, C. M. (1974), Migration enhancement factor: a new lymphokine. Proc. Natl. Acad. Sci. 71, 875-879. doi: 10.1073/pnas.71.3.875

Weissleder, R., Nahrendorf, M., and Pittet, M. J. (2014). Imaging macrophages with nanoparticles. Nat. Mater. 13, 125-138. doi: 10.1038/nmat3780

Xie, J., Lee, S., and Chen, X. (2010). Nanoparticle-based theranostic agents. Adv. Drug Deliv. Rev. 62, 1064-1079. doi: 10.1016/j.addr.2010.07.009

Yordanova, A., Eppard, E., Kürpig, S., Bundschuh, R. A., Schönberger, S., Gonzalez-Carmona, M., et al. (2017). Theranostics in nuclear medicine practice. Onco. Targets. Ther. 10:4821. doi: 10.2147/OTT.S140671

Zavaleta, C., Ho, D., and Chung, E. J. (2018). Theranostic nanoparticles for tracking and monitoring disease state. SLAS Technol. 2018:2472630317738699. doi: 10.1177/2472630317738699

Zhang, J., Zu, Y., Dhanasekara, C. S., Li, J., Wu, D., Fan, Z., et al. (2017). Detection and treatment of atherosclerosis using nanoparticles. Wiley Interdiscip. Rev. Nanomed. Nanobiotechnol. 9:1412. doi: 10.1002/wnan.1412

Conflict of Interest Statement: The authors declare that the research was conducted in the absence of any commercial or financial relationships that could be construed as a potential conflict of interest.

Copyright (c) 2018 Cervadoro, Palomba, Vergaro, Cecchi, Menichetti, Decuzzi, Emdin and Luin. This is an open-access article distributed under the terms of the Creative Commons Attribution License (CC BY). The use, distribution or reproduction in other forums is permitted, provided the original author(s) and the copyright owner(s) are credited and that the original publication in this journal is cited, in accordance with accepted academic practice. No use, distribution or reproduction is permitted which does not comply with these terms. 